\title{
Percolation in Systems Containing Ordered Elongated Objects
}

\author{
P. Romiszowski* ${ }^{*}$ A. Sikorski \\ Department of Chemistry, University of Warsaw \\ Pasteura 1, 02-093 Warsaw, Poland \\ *E-mail: prom@chem.uw.edu.pl
}

Received: 21 February 2013; revised: 13 April 2013; accepted: 16 April 2013; published online: 22 May 2013

\begin{abstract}
We studied the percolation and jamming of elongated objects using the Random Sequential Adsorption (RSA) technique. The objects were represented by linear sequences of beads forming needles. The positions of the beads were restricted to vertices of two-dimensional square lattice. The external field that imposed ordering of the objects was introduced into the model. The percolation and the jamming thresholds were determined for all systems under consideration. The influence of the chain length and the ordering on both thresholds was calculated and discussed. It was shown that for a strongly ordered system containing needles the ratio of percolation and jamming thresholds $c_{p} / c_{j}$ is almost independent on the needle length $d$.
\end{abstract}

Key words: percolation, Monte Carlo method, random sequential adsorption, jamming

\section{INTRODUCTION}

The process of percolation in complex molecular systems attracts researchers' attention for its importance in porous media, alloys and composites [1]. Percolation can occur in two-component systems, e.g. polymer-solvent. From the point of view of one component a site on a substrate is occupied or non-occupied. Neighboring objects form a cluster and if this cluster becomes infinitely spanned (in the case of simulations this cluster is finite but it spans from one border of the system to another) then the percolation occurs in the system. The theory of percolation has many applications in physics, especially in disordered systems and porous media. The percolation phenomena in systems containing carbon nanotubes were studied experimentally. In systems containing composites of carbon nanotubes and liquid crystals the percolation process was studied by the measurements of electrical conductivity [2]. Recent development in nanotubes and block copolymers containing styrene derivatives show that the mixing conditions have an important influence on the conductivity of carbon natonube polymer composites [3]. The critical behavior of long straight rods was discussed in the paper of Nieto and coworkers who studied the transition between ordered and isotropic phases [4-7].

The most popular method of studying the percolation process is the Random Sequential Adsorption (RSA) technique [8-9]. In this process objects are placed subsequently on a substrate and then immobilized; the overlapping of objects was forbidden. An alternative methodology is based on the classical Metropolis scheme: the system contained the objects at a given density and their configuration was modified and the probability of percolation was determined $[10,11]$.

The non-spherical objects were also studied by the RSA method [8-9, 12-18] and in theoretical considerations [19-23]. The lattice representation of the studied objects was applied much more frequently. Discretization of the space enables one to study considerably larger systems at higher time scale. It was shown that the percolation threshold in such systems did not change monotonically with the length of needles. For short objects the threshold decreases rapidly and then it started to increase moderately. This non-monotonic behavior of the percolation threshold is quite different from that of nonlinear objects (T-shaped and crosses), where the decrease of the threshold occurs for all sizes of objects. Introduction of 
flexibility into linear elongated objects which can be treated as a model of polymer chains did not change qualitatively the behavior of the percolation threshold but shifted the threshold towards slightly higher densities. The behavior of the jamming threshold differed for flexible chains significantly as decreased with the chain length very rapidly. It was also shown that contrary to other non-linear objects local ordering had an impact on the percolation and jamming of systems containing needles: highly ordered clusters of needles were observed.

When dealing with stiff elongated objects (needles) the question appears what happens to the percolation and jamming when the probability of taking horizontal and vertical orientation is not the same. In other words, is the influence of the global ordering of the system on percolation and jamming? It was recently shown that in the case of dimers percolation had the maximum value for non-aligned systems [24]. The problem of percolation of ordered systems is strongly connected to the phenomenon of the nematic-disordered phase transition which was studied by Ramirez-Pastor and coworkers [6-7] and Ghosh and Dhar [25]. The authors found that for the rigid rods of length $k \geq 7$ one observes nematicdisordered phase transition depending on the density of the system. The recent paper of Kählitz and Stark [26] presents the simulations of hard needles on a quasi-crystalline substrate. They found that the size of the oriented domains depends on the length of the needles. The phase diagrams presenting the nematic ordering for short and long needles are presented. The structure of the systems containing needles in the external field was recently studied by Tarasevitch et al. $[27,28]$. They introduced a new simulation method - the Random Relaxation Sequential Adsorption (RRSA). The authors presented the percolation study of linear k-mers forming the structures of different ordering, from isotropic to completely ordered states, and proposed the fitting formula for the percolation threshold depending on anisotropy of the system.

In this work we present a study of the percolation in systems containing extended objects (needles) located in an ordering external field. The aim of this study was to investigate a structure of strongly adsorbed polymer films and therefore we used a 2-dimensional model. In the 2-dimensional model the excluded volume effect strongly influences the properties of the objects under consideration. The lattice representation of objects was employed in order to speed up the calculations. The RSA method was applied to determine both percolation and jamming thresholds.

\section{THE MODEL AND THE SIMULATION METHOD}

The objects used in our simulations were linear sequences of beads forming a rod (needle). Each needle consisted of $d=2,5,10,20,40,60,80$ or 100 beads. The simulations were carried out for the Monte Carlo box the square lattice $L \times L$ with $L=50,100,200,500$ and 1000 lattice units with hard boundary conditions (HBC). The simulations were performed using the Random Sequential Adsorption (RSA) procedure [8-9]. As we mentioned in the previous chapter, the RRSA method results from the final distribution of the orientation of object that is the same as assumed. In the RSA method the final distribution of the orientation of the objects differs from the assumed one since the presence of the already deposited needles block the free sites. The differences between these two methods can be interpreted as the strength of binding. In our RSA method the basic requirement of adding the objects on the substrate was the presence of an excluded volume effect which prohibited any intersection of the added needle with any other object placed previously. The second condition was an irreversible adsorption, i.e. the added objects stayed in their positions to the end of the process and their orientations also remained unchanged during the simulation. The objects were subsequently added to the system at a random knot of the lattice and placed in one of the allowed directions: horizontally or vertically with respect to the $x$ axis. The orientations were taken with the probability $\varepsilon$, which was varied from 0 to 1 and was constant at given simulation run, which enabled us to obtain the fully oriented system (for $\varepsilon=1$ or 0 ) or the random placement of objects ( $\varepsilon=0.5)$, respectively. The ordering parameter $\varepsilon$ played a role of an external field which was present during the simulation run and thus enabled us to determine the effect of the field strength on the percolation of the system. The process of adding objects was carried out up to the stage in which adding a needle to the system was not possible because all possible free sites were occupied, i.e. the jamming threshold $c_{j}$ was reached. The end of simulation was assumed when in 100 consecutive cycles no new object could be added to the system. The percolation threshold $c_{p}$, which was obviously smaller than $c_{j}$ was also determined during the course of the simulation using an algorithm based on the HoshenKopelman scheme [18, 29]. In order to check the repeatability of the calculation method and to minimize the fluctuations in simulation results we performed from 100 (large systems) to 1000 (small systems) simulation runs for each set of data and then the results were averaged. In order to determine the percolation threshold for the infinite system a scaling analysis was performed: the values of the threshold were extrapolated according to the scaling law

$$
\left|c_{p}(L)-c_{p}(\infty)\right| \sim L^{-1 / \nu},
$$

where $c_{p}(L)$ and $c_{p}(\infty)$ are percolation thresholds for the Monte Carlo box $L \times L$ and for an infinite system, respectively, and $n$ is a critical exponent (theoretical predictions give $n=4 / 3$ ) [1]. A finite size scaling analysis according to Eq. (1) was done. In the next section we will discuss the results determined for the infinite system. The universality of Eq. 1 has been confirmed since it is obeyed for all length of needles we used. The standard error of the thresholds was of order 0.001 . 


\section{RESULTS AND DISCUSSION}

In order to investigate the results of the simulations we analyzed the data showing the percolation thresholds $c_{p}$ and jamming thresholds $c_{j}$ for each set of input data such as the length of the needles, size of the system and orientation parameter $\varepsilon$. In order to present the relation between the percolation threshold $c_{p}$ and the order parameter we introduced the order parameter $P_{2}$, which is the second-rank orientation order parameter in two dimensions and was defined as

$$
P_{2}=\left\langle 2 \cos ^{2} \Theta-1\right\rangle,
$$

where $q$ is the angle between a needle and the $y$ axis. The averaging was performed over all objects and all simulation runs. In the case of a square lattice and the only two possible orientations of needles are only allowed $(q=0$ or $q=p / 2)$ and therefore the relation (2) can be reduced to

$$
P_{2}=\left(N_{\mid}-N_{-}\right) /\left(N_{\mid}+N_{-}\right),
$$

where $N_{\mid}$and $N_{-}$are the numbers of needles oriented vertically and horizontally with respect to the $x$ axis.

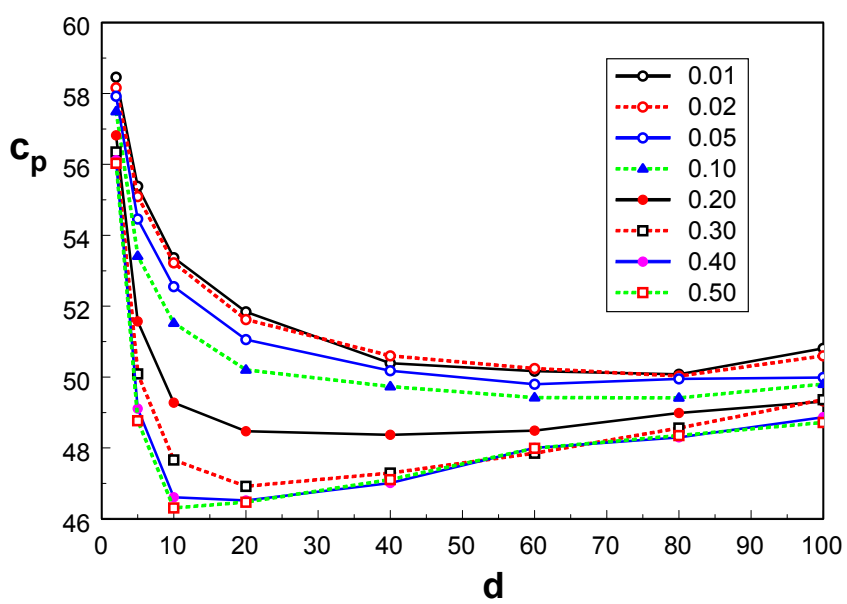

Fig. 1. The percolation threshold $c_{p}$ as a function of the length of needle $d$. The values of the order parameter are given in the inset

Figure 1 presents the values of the percolation thresholds $c_{p}$ as a function of the length of the needles $d$. Each curve was obtained from the simulations performed at different values of $\varepsilon$. The presented plots form a family of curves which have the common course - as the needle length increases the values of $c_{p}$ decreases and after that stage increases depending on the values of the ordering parameter. The curve obtained for $\varepsilon=0.5$ shows the fully random orientation of needles and therefore can be a reference for the systems which are formed in an orienting external field. One can notice that the percolation threshold decreases along with the increase of the needle length with the exception of cases for vertical needles $\varepsilon \geq 0.2$ for which one can observe the minimum on the curves. The influence of the ordering for short needles is the strongest for the intermediate length of chain $(d=5$ to 40$)$ with the exception for dimers $(d=2)$ where the influence of ordering is rather small and the percolation threshold is within the range $c_{p}=$ from 0.56 to 0.58 . The changes of $c_{p}$ for dimers is almost the same as found recently by Ramirez-Pastor et al. [30]. It is also interesting that $c_{p}$ is more sensitive on the field strength in its intermediate values ( 0.1 to 0.3$)$ where it changes the percolation threshold the most. This effect can be explained that for the random orientations of needles the elongation of the objects blocks certain areas making then inaccessible for adding new needles via the RSA process.

In Figure 2 we present the changes of the jamming threshold $c_{j}$ as a function of length of needles $d$ at given orientation parameter $\varepsilon$. One can notice that similarly to the percolation threshold for stronger ordering the jamming threshold is larger than for the randomly positioned rods, i.e. for $\varepsilon=0$. One can also observe that the curves are almost parallel and exhibit strong decrease of $c_{j}$ for short objects (up to $d=10$ ) with the length of objects. This effect does not depend on the strength of the ordering field $\varepsilon$ since short objects can be placed in both directions forming the relatively dense structure, while for longer objects $(d>20)$ the presence of the strong ordering field leads to higher values of $c_{j}$ comparing it with more random positioning of rods mutually blocking the positions, which leads to more empty sites in a jammed system. One can notice that for stronger ordering of the system the jamming threshold values increase because the ordered system can adsorb more objects which form parallel structures than the random one.

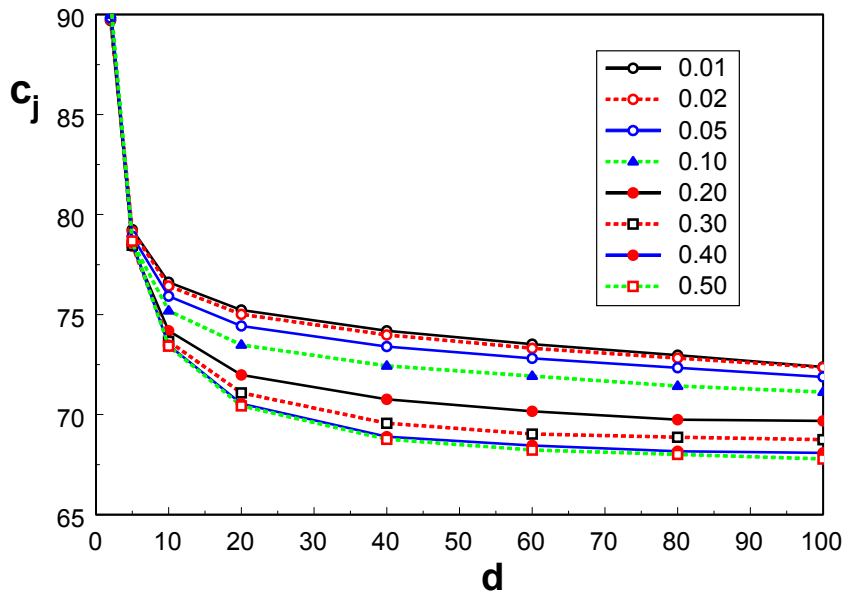

Fig. 2. The jamming threshold $c_{j}$ as a function of the length of needle $d$. The values of the order parameter are given in the inset 


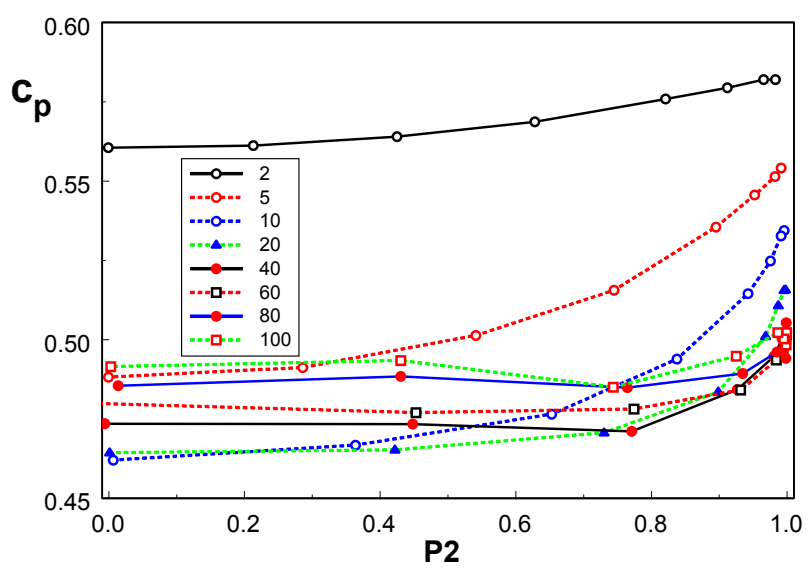

Fig. 3. The percolation threshold $c_{p}$ as a function of the order parameter $P_{2}$. The lengths of needles are given in the inset

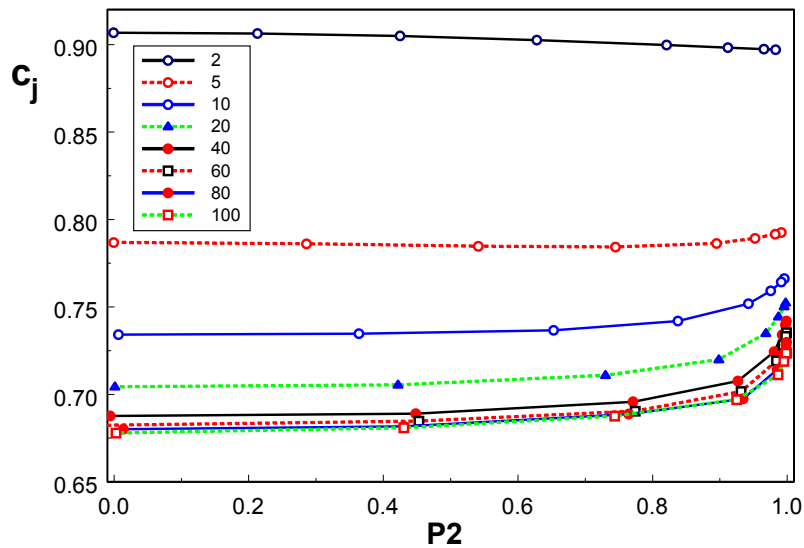

Fig. 4. The jamming threshold $c_{j}$ as a function of the order parameter $P_{2}$. The lengths of needles are given in the inset

The effect of ordering the system on both the percolation and jamming thresholds for different rod length $d$ is presented in Figures 3 and 4. The percolation threshold increases along with the increase of order parameter $P_{2}$ for shorter needles (up to $d=20$ ), while for longer objects the curves show shallow minima at vicinity of $P_{2}=0.8$ and then the increase of $c_{p}$ as $P_{2}$ approaches 1 . One can notice that the curve for dimers shows a weak dependence on $P_{2}$ comparing that tendency for longer chains, which is especially visible for strong ordering. This result was reported for the percolation threshold $c_{p}$ for dimers by Tarasevich and coworkers who compared the values of fully ordered and disordered systems [28]. The jamming threshold for dimers exhibits a slow decrease when the ordering in the system becomes larger while for all longer needles $(d \geq 5) c_{j}$ values increase slightly with the ordering. However, for high ordering $\left(P_{2}>0.8\right)$ this increase becomes more rapid. Comparing both figures one can notice that the percolation threshold depends more on the order parameter than the jamming threshold does, and this effect is more pronounced for medium length needles $(5 \geq d \geq 20)$. These results are in agreement with previous findings for dimers [28]. One should also notice that the case of dimers $(d=2)$ is much less sensitive on the ordering than longer objects, which shows that dimers form relatively dense jammed systems almost independently of the value of ordering field. This is evident if one realizes that empty space left by oriented or disordered dimers does not differ very much since the small size of the objects enables achieving a dense packing while in the case of longer needles the presence of empty space is more pronounced. One should notice that the results of Tarasevich et al. [28] are very close to our findings despite the fact that we used a different simulation method (RSA vs. RRSA). This is not a surprise while the percolation of the system is reached for relatively low densities for which the insertion of a new needle to the system does not need attempts. The differences between our results for jamming threshold and the findings of Tarasevitch et al. [27] is more pronounced for longer and strongly oriented needles.

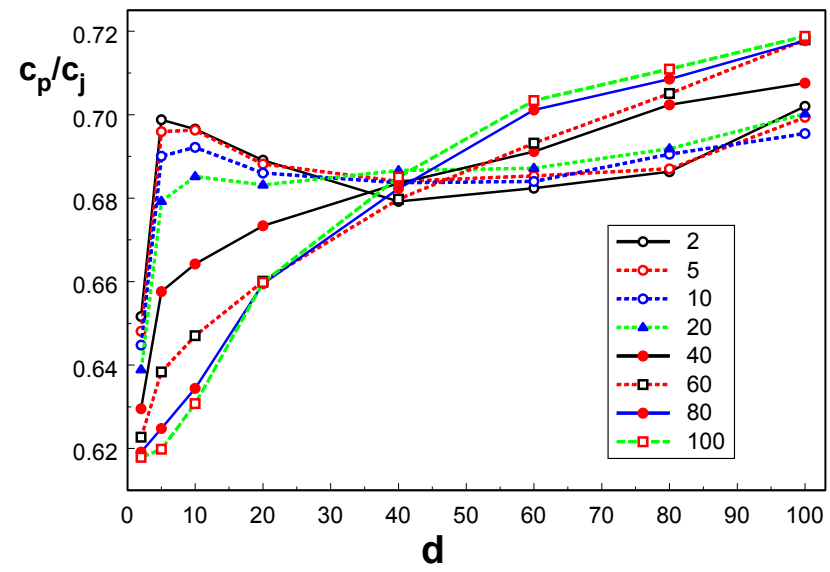

Fig. 5. The ratio $c_{p} / c_{j}$ as a function of the length of needle $d$. The values of the order parameter are given in the inset

The ratio of both thresholds $c_{p} / c_{j}$ is widely used as a parameter that describes the structure of adsorbed objects. In our study we plotted $c_{p} / c_{j}$ as a function of both the rod length $d$ (Figure 5) and order parameter $P_{2}$ (Figure 6). Figure 5 presents the dependence of the ratio $c_{p} / c_{j}$ as a function of object length $d$ for different field strength. One can notice that for a strong ordering field the $c_{p} / c_{j}$ increases rapidly for short needles and then it becomes rather stable at level $0.68-0.70$ with slight growth for long objects. This shows that for strongly ordered objects the properties of the systems scale almost independently of the length of objects $d-$ the parallel objects have similar properties since the long needle can be treated (to some extent) as the sum of shorter needles. The weaker field $(0.3 \geq \varepsilon \geq 0.5)$ shows quite different behavior: the ratio $c_{p} / c_{j}$ increases gradually from 0.62 to almost 0.72 along the whole range of object length used in the simulations. 


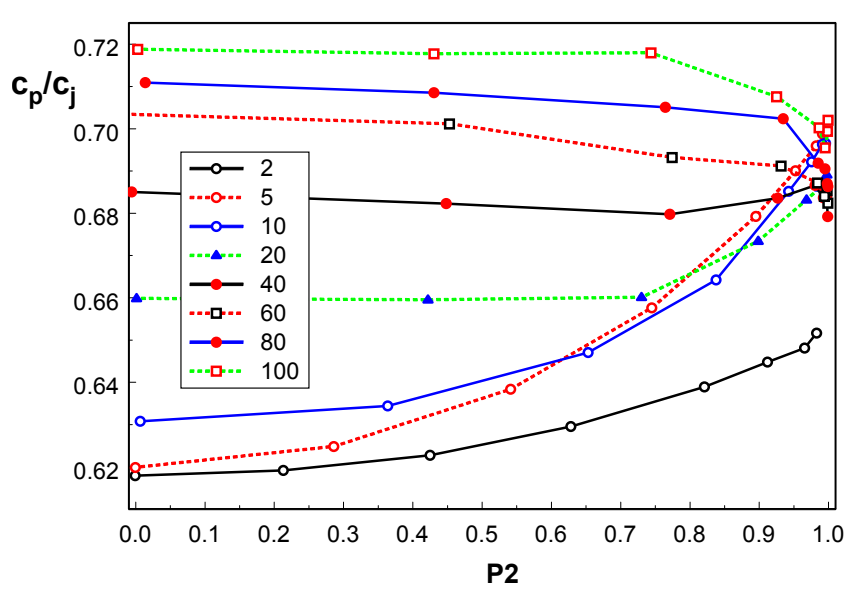

Fig. 6. The ratio $c_{p} / c_{j}$ as a function of the order parameter $P_{2}$. The lengths of needles are given in the inset

An interesting observation is that there is a common region of intersection of all curves at $d \approx 40$. The relation between $c_{p} / c_{j}$ and $P_{2}$ plotted in Figure 6 shows that for strongly ordered systems the $c_{p} / c_{j}$ ratio goes to the vicinity of $c a .0 .7$ independently of the rod length - some curves for longer needles decrease while those for shorter rods increase along with the order parameter. This effect is the consequence of the approximation which enables one to treat the long parallel needles as the sum of shorter objects resulting from the proximity of points in a common plot. One can observe that the curve for $d=40$ is almost independent on $P_{2}$ which is shown in Figure 5 as one common region of intersections of all curves. This effect can be explained if one notices that for shorter needles the plots $c_{p} / c_{j}$ vs. $P_{2}$ increase while for longer needles they decrease along with the values of $P_{2}$ - in such case there must be a curve which is almost independent on $P_{2}$. The results concerning the ratio $c_{p} / c_{j}$ which were obtained for the systems without the ordering field $(e=0)$ are close to those obtained by Vandewalle et al. [14] where this parameter fluctuated near 0.62 for short needles $(d \geq 10)$. We do not confirm the results obtained by Tarasevitch et al. [28] where the strongly ordered systems exibit a plateau in the ratio $c_{p} / c_{j}$ for longer neeedles $(d>8)$.

In Figures 7-8 we present typical snapshots of the system under consideration showing the structure of adsorbed needles at the percolation threshold. Figures 7a-c show the snapshots of the percolation of a system of short needles $(d=10)$ at different values of the ordering field strength $\varepsilon=0.05,0.20$ and 0.50 , respectively. As one can notice, the percolation cluster became larger as the rods were less ordered. The set of Figures 8a-c shows the changes in percolation clusters as the length of the needles increased for $d=5$ (a), 20 (b) and 80 (c) lattice units at the same moderate value of ordering parameter $\varepsilon=0.30$, respectively. One can notice that the shorter the objects, the more complicated fractal structure of the percolation cluster was obtained. As the length of needles increases a noticeable number of vacancies in the system can be observed. This is in full agreement with the data plotted in Figure 2.

\section{CONCLUSIONS}

The lattice approximation of elongated objects was used in order to simulate the Random Sequential Adsorption (RSA) process. The parameters that characterized the systems under consideration such as the percolation and jamming thresholds as well as the order parameter of the resulting systems were determined. The finite size scaling analysis was done in order

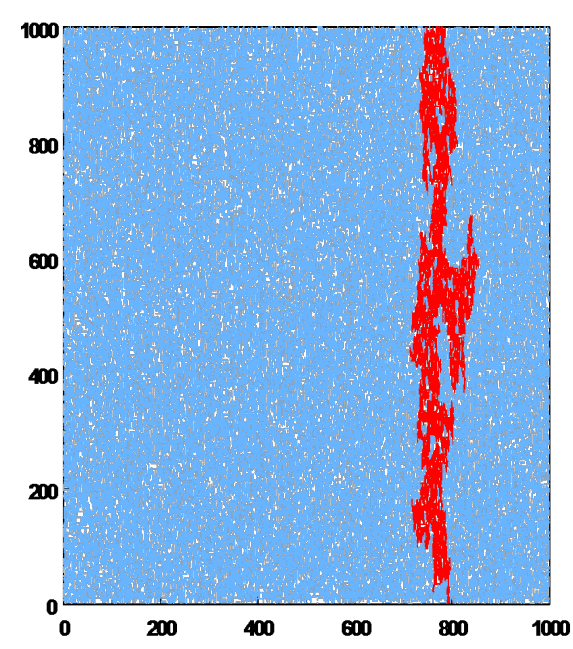

(a)

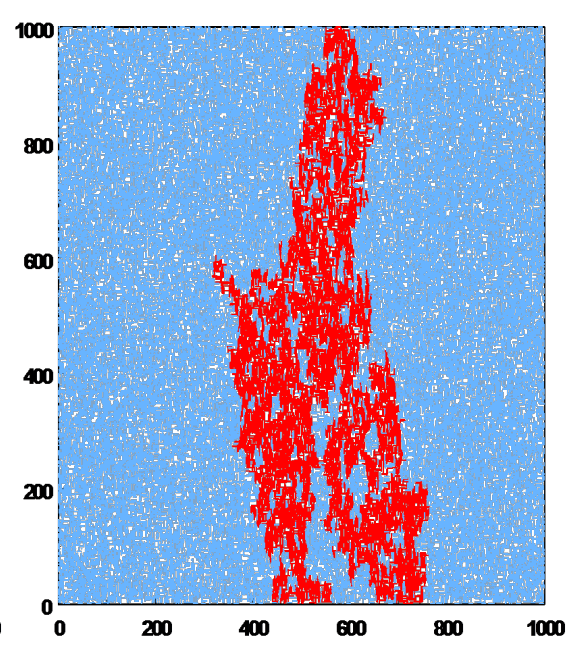

(b)

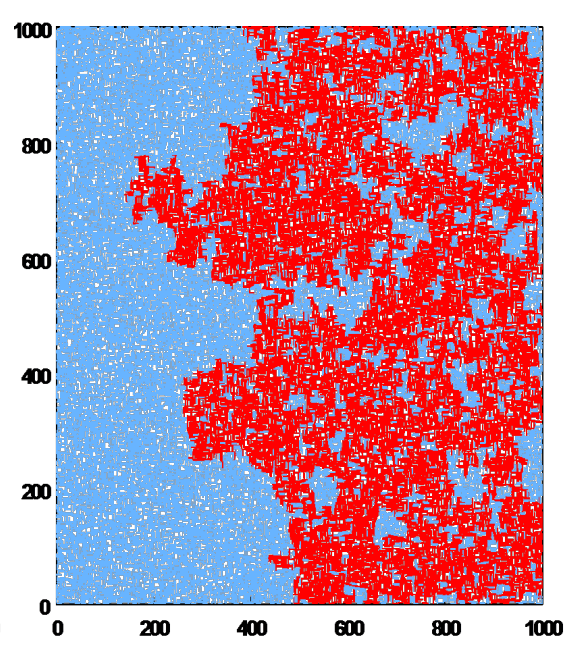

(c)

Fig. 7. The snapshots of the system containing needles $d=10$ at the percolation threshold for the ordering field $\varepsilon=0.05$ (a), 0.10 (b) and $0.50(\mathrm{c})$, respectively. The percolation clusters are marked in red, while all other clusters are colored in blue 


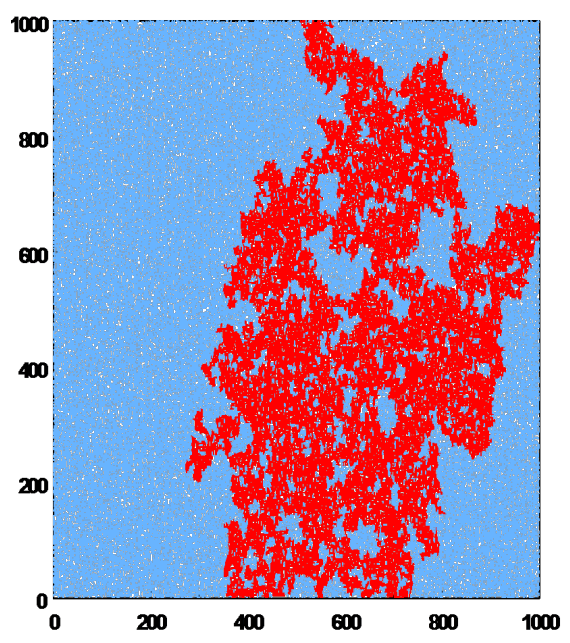

(a)

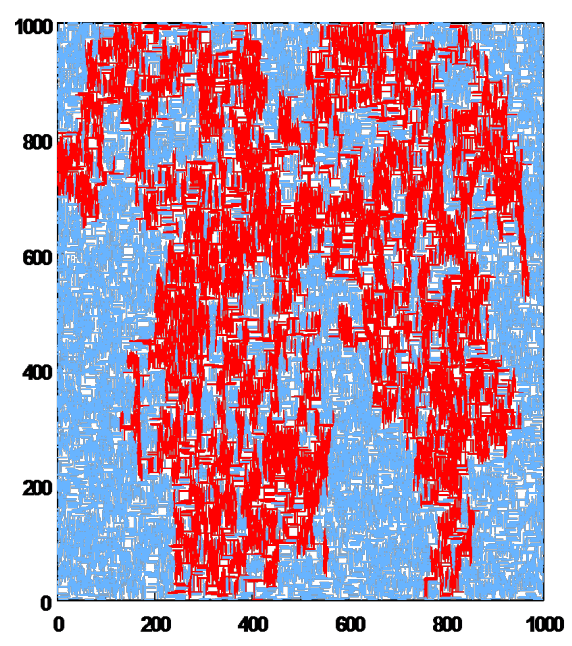

(b)

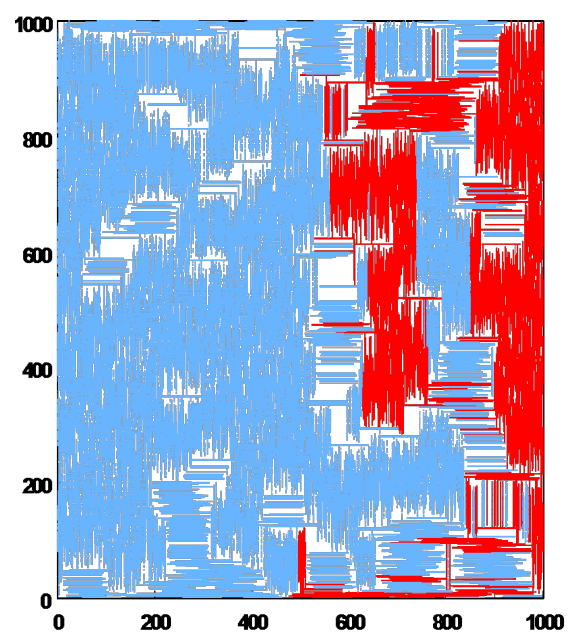

(c)

Fig. 8. The snapshots of the system at the percolation thresholds for needles $d=5$ (a), 20 (b) and 80 (c) for the ordering parameter $\varepsilon=0.30$. The percolation clusters are marked in red, while all other clusters are colored in blue

to estimate the calculated parameters for the infinite system.

It was shown that the presence of ordering field $\varepsilon$ changes the values of $c_{p}$ and $c_{j}$ but this influence depends not only on the field's strength but also on the length of the rods. For short as well as long rods the system is less sensitive to the presence of ordering field while for intermediate rod length ( $d=10$ to 40 ) the changes of percolation threshold $c_{p}$ are more pronounced.

\section{Acknowledgment}

The computational part of this work was done using the computer cluster at the Computing Center of the Department of Chemistry, University of Warsaw. This work was partially supported by the Polish Ministry of Science and Higher Education grant N N204 118539.

\section{References}

[1] D. Stauffer, A. Aharony, Introduction to Percolation Theory (Taylor and Francis, London 1994).

[2] L.N. Lisetski, S.S. Minenko, A.P. Fedoryako and N.I. Lebovka, Dispersions of multiwalled carbon nanotubes in different nematic mesogens: The study of optical transmittance and electrical conductivity, Physica E 41, 431 (2009).

[3] J.G. Meier, C. Crespo, J.L. Pelegay, P. Castell, R. Sainz, W.K. Maser, A.M. Benito, Processing dependency of percolation threshold of MWCNTs in a thermoplastic elastomeric block copolymer, Polymer 52, 1788 (2011).

[4] Matoz-Fernandes, D.H. Linares, A.J. Ramirez-Pastor, Europhys.Lett., Determination of the critical exponents for the isotropic-nematic phase transition in a system of long rods on two-dimensional lattices: Universality of the transition, Europhys.Lett. 82, 50007 (2008).

[5] L.G. Lopez, D.H. Linares, A.J. Ramirez-Pastor, S.A. Cannas, Phase diagram of self-assembled rigid rods on twodimensional lattices: Theory and Monte Carlo simulations, J. Chem. Phys. 133, 134706 (2010).
[6] V. Cornette, A.J. Ramirez-Pastor, F. Nieto, Dependence of the percolation threshold on the size of the percolating species, Physica A 327, 71 (2003).

[7] V. Cornette, A.J. Ramirez-Pastor, F. Nieto, Percolation of polyatomic species on a square lattice, Eur. Phys. J. B 36, 391 (2003).

[8] J.W. Evans, Random and cooperative sequential adsorption, Rev. Mod. Phys. 65, 1281 (1993).

[9] J. Talbot, G. Tarjus, P.R. Van Tassel, P. Viot, From car parking to protein adsorption: an overview of sequential adsorption processes, Colloid. Surface A 165, 287 (2000).

[10] P. Adamczyk, P. Polanowski, A. Sikorski, Percolation in polymer-solvent systems: A Monte Carlo study, J. Chem. Phys. 131, 234901 (2009).

[11] M. Pawłowska, S. Żerko, A. Sikorski, Note: Percolation in two-dimensional flexible chains systems, J. Chem. Phys. 136, 046101 (2012).

[12] R.D. Vigil, R.M. Ziff, Random sequential adsorption of unoriented rectangles onto a plane, J. Chem. Phys. 91, 2599 (1989).

[13] R.M. Ziff, R.D. Vigil, Kinetics and fractal properties of the random sequential adsorption of line segments, J. Phys. A: Math. Gen. 23, 5103 (1990).

[14] N. Vandewalle, S. Galam, M. Kramer, A new universality for random sequential deposition of needles, Eur. Phys. J. B 14, 407 (2000).

[15] G. Kondrat, A. Pekalski, Percolation and jamming in random sequential adsorption of linear segments on a square lattice,Phys. Rev. E 63, 051108 (2001).

[16] G. Kondrat, A. Pekalski, Percolation and jamming in random bond deposition, Phys. Rev. E 64, 056118 (2001).

[17] G. Kondrat, Influence of temperature on percolation in a simple model of flexible chains adsorption, J. Chem. Phys. 117, 6662 (2002).

[18] P. Adamczyk, P. Romiszowski, A. Sikorski, A simple model of stiff and flexible polymer chain adsorption: The influence of the internal chain architecture, J. Chem. Phys. 128, 154911 (2008).

[19] E.J. Garboczi, K.A. Snyder, J.F. Douglas, M.F. Thorpe, Geometrical percolation threshold of overlapping ellipsoids, Phys. Rev. E 52, 819 (1995). 
[20] J.-S. Wang, Series expansion and computer simulation studies of random sequential adsorption, Colloid. Surface A $\mathbf{1 6 5}$, 325 (2000).

[21] X. Wang, A.P. Chatterjee, Connectedness percolation in athermal mixtures of flexible and rigid macromolecules: Analytic theory, J. Chem. Phys. 118, 10787 (2003).

[22] Y.B. Yi, A.M. Sastry, Analytical approximation of the percolation threshold for overlapping ellipsoids of revolution, Proc. Royal Soc. Lond. A 460, 2353 (2004).

[23] A. P. Chatterjee, Percolation thresholds for rod-like particles: polydispersity effects, J. Phys.: Condens. Matter 20, 255250 (2008).

[24] V.A. Cherkasova, Y.Y. Tarasevich, N.I. Lebovka, N.V. Vygornitskij, Percolation of aligned dimers on a square lattice, Eur. Phys. J. B 74, 205 (2010).

[25] N.I. Lebovka, N.N. Karmazina, Y.Yu. Tarasevich, V.V. Laptev, Random sequential adsorption of partially oriented linear $k$-mers on a square lattice, Phys. Rev. E, 85, 029902 (2012).

[26] A. Ghosh, D. Dhar, On the orientational ordering of long rods on a lattice, Eur. Phys. Lett. 78, 20003 (2007).
[27] P.Kählitz, H.Stark, Phase ordering of hard needles on a quasicrystalline substrate, J.Chem.Phys. 136, 174705 (2012).

[28] Y.Yu. Tarasevich, N.I.Lebovka, V.V. Laptev, Percolation of linear $k$-mers on a square lattice: From isotropic through partially ordered to completely aligned states, Phys. Rev. E 86, 061116 (2012).

[29] J. Hoshen, R. Kopelman, Percolation and cluster distribution. I. Cluster multiple labeling technique and critical concentration algorithm, Phys. Rev. B 14, 3438 (1976).

[30] M. Dolz, F. Nieto, A.J. Ramirez-Pastor, Dimer site-bond percolation on a square lattice, Eur. Phys. J. B 43, 363 (2005).
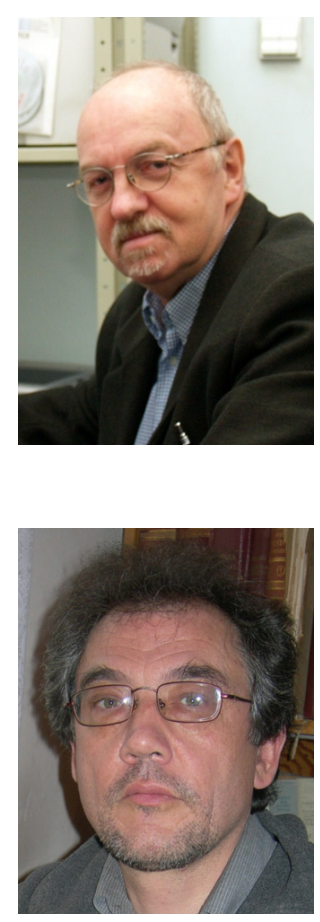

Piotr Romiszowski graduated from the Department of Chemistry, University of Warsaw in 1970. He completed his PhD in the same Department in 1979. He was a Fulbright Fellow at Dartmouth College (1982/83) and a post-doc at Washington University in St. Louis, Missouri (1989/91). He is interested in the theory and simulation of polymers in confined systems and at interfaces.

Andrzej Sikorski graduated from the Department of Chemistry, University of Warsaw in 1980. He completed his PhD Thesis in Chemistry in 1986 in the same Department. In 1987/89 and 1990/91 he worked as a postdoc and visiting professor in Washington University in St. Louis and in The Scripps Research Institute in La Jolla. In 1995 he obtained DSc degree in Chemistry. His main scientific interest is the theory and simulation of polymers at interfaces and in confined geometries. 